\title{
EXPERIMENTAL STUDIES \\ OF ELECTRONIC PHASE SEPARATION IN HIGH TEMPERATURE SUPERCONDUCTORS
}

\author{
H. SZYMCZAK AND R. SZYMCZAK \\ Institute of Physics, Polish Academy of Sciences \\ Al. Lotników 32/46, 02-668 Warszawa, Poland
}

\begin{abstract}
Experimental research on macroscopic phase separation in $\mathrm{La}_{2} \mathrm{CuO}_{4+\delta}$ superconductor and photo-doped cuprates is reviewed. It is shown that the properties of the mixed-phase state strongly depend on the shape and size of the phase domains formed during the phase separation process. The interplay of magnetic and superconducting properties in the mixed-phase state is considered in details. The mechanisms driving the phase separation in $\mathrm{La}_{2} \mathrm{CuO}_{4}$ and photo-doped materials are discussed in connection with presented experimental results.
\end{abstract}

PACS numbers: 74.25.Ha, 74.72.-h

\section{Introduction}

Although enormous effort has been made into investigations of the high- $T_{\mathrm{c}}$ superconductors since their discovery in 1986 [1], many aspects of this phenomenon remain unexplained, including a fundamental problem of the mechanisms responsible for this effect. Nevertheless, several characteristic features of high- $T_{\mathrm{c}}$ cuprates have been found. A central feature of this group of superconducting materials is that they are doped insulators, obtained by chemical doping (realized by mixed valence substitution, as in $\mathrm{La}_{2-x} \mathrm{Sr}_{x} \mathrm{CuO}_{4}$, or by variation of oxygen content, as in $\mathrm{YBa}_{2} \mathrm{Cu}_{3} \mathrm{O}_{7-x}$ ) or by photoexcitation. The starting material is always an insulating antiferromagnet with the rather high (200-300 K) ordering temperature $T_{\mathrm{N}}$ (Néel temperature). The ordering temperature of the parent material decreases rapidly with increasing doping proceeding to a magnetically disordered state and finally to a conducting state which becomes superconducting below the critical temperature $T_{\mathrm{c}}$.

Usually it is assumed that the doping leads to a homogeneous electronic (conducting) state. But it has been already realized that under some conditions antiferromagnetism will become unstable upon doping. Such a possibility has been considered for the first time by Vischer [2]. He has shown in terms of the Hubbard model that in antiferromagnets upon doping the phase separation appears. The 
system is divided into spatially separated regions of nearly neutral antiferromagnetic order and into ferromagnetic regions of high carrier concentration. In the case of the two-dimensional antiferromagnet it has been suggested that dilute holes are unstable against the phase separation into a hole-rich, metallic phase and a pure antiferromagnetic insulating phase [3-5]. In high- $T_{\mathrm{c}}$ superconductors the macroscopic phase separation has been observed only in oxygen-doped $\mathrm{La}_{2} \mathrm{CuO}_{4}[6]$ and in photo-doped materials (see [7] and [8] for references).

In the majority of high-temperature superconductors the dopant atoms are frozen at ambient temperature and therefore the phase separation can only take place as a short distance, fluctuation effect (microscopic phase separation). On the contrary, the excess oxygen atoms in $\mathrm{La}_{2} \mathrm{CuO}_{4+\delta}$ are mobile and easily migrate above $280 \mathrm{~K}$ that results in the phase separation. It was assumed, until now, that the phase separation was the direct consequence of high enough oxygen mobility in the system. Recently, experiments performed by Kremer et al. [9, 10] questioned in part this common opinion. From the experimental findings it has been confirmed [9], without any doubt, that the observed phase separation is a diffusion controlled process. The problem concerns the particles which can carry out diffusive motions. These are individual charge carriers (holes) forming small spin-polarized clusters [11] or excess oxygen ions. It is suggested [10] that although both types of diffusional motion exist in $\mathrm{La}_{2} \mathrm{CuO}_{4+\delta}$, the hole motion in the temperatures above $150 \mathrm{~K}$ essentially contributes to the diffusion process and to the phase separation. They additionally suggest that the oxygen diffusion is not essential for the formation of the superconducting subphase. Depending on the experimental conditions the motion of the oxygen atoms not only supports but can also suppress this phase formation. It seems that the role of the oxygen mobility in formation of the macroscopically separated phases is not finally clarified. It is known [9] for example that in the $\mathrm{La}_{2-x} \mathrm{Sr}_{x} \mathrm{CuO}_{4}$ system the macroscopic phase separation has not been observed. This observation is directly connected with the fact that diffusion of $\mathrm{Sr}$ ions at the relevant temperature is negligibly small. Therefore only small conducting microphases can be formed in this system [12]. In order to show the effect of a microscopic segregation, Cho et al. [12] performed high resolution in $x$ studies of the magnetic and structural phase diagram of $\mathrm{La}_{2-x} \mathrm{Sr}_{x} \mathrm{CuO}_{4+\delta}$ in the low doping regime $0 \leq(x, \delta) \leq 0.03$, where the doped holes were produced by Sr doping and/or doping with excess oxygen. They observed that for $\delta=0$ (no mobile oxygen) $T_{\mathrm{N}}(x)$ is decreasing extremely rapidly from about $300 \mathrm{~K}$ for $x=0$ to $0 \mathrm{~K}$ by $x_{\mathrm{N}} \simeq 0.02$. In the oxygen doped material $(\delta>0), T_{\mathrm{N}}(x, \delta)$ and $T_{\mathrm{c}}(x, \delta)$ are all diminished by Sr doping, namely: $T_{\mathrm{N}}(x, \delta)$ continues to vanish at about $x \simeq x_{\mathrm{N}}$, whereas superconductivity is observed up to $x \simeq 0.03$. Quantitative analysis of the experimental data [12] for the antiferromagnetic phase indicates that $T_{\mathrm{N}}(x)$ and magnetic susceptibility $\chi(x, T)$ are determined by finite size effects induced by doping. The doped holes form hole-rich walls separating microscopic domains of undoped materials. All of the reviewed data concerning $\mathrm{La}_{2-x} \mathrm{Sr}_{x} \mathrm{CuO}_{4}$ evidence that the microscopic segregation in this material is electronically driven. The presence of immobile Sr dopants implies a minimum hole concentration in the hole-poor-domains reducing the antiferromagnetic correlations in the $\mathrm{CuO}_{2}$ planes, responsible for both phase separation and magnetic ordering. 
Another mechanism of the phase separation, the so-called "chemical mechanism" [13], is based on the lattice forces leading to clustering of ions and atoms into large aggregates. As it was mentioned, the chemically driven phase separation directly related to oxygen mobility, has been observed in the experiment performed by Kremer et al. [9] on oxygenated $\mathrm{La}_{2} \mathrm{CuO}_{4+\delta}$. Even stronger experimental evidence that the phase separation in oxygenated $\mathrm{La}_{2} \mathrm{CuO}_{4+\delta}$ is driven, at least partly, by superfluous oxygen chemistry, has been reported by Ahrens et al. [14]. They have investigated the effect of cooling rate on $T_{\mathrm{c}}$ and microstructure in super-oxygenated $\mathrm{La}_{2} \mathrm{CuO}_{4+\delta}$. They show strong sensitivity to the cooling rate depending on whether the sample is quenched or slow cooled through a narrow temperature range around $(195 \pm 10) \mathrm{K}$. This result correlates with the measured [15] temperature dependence of the excess oxygen content in the insulating and metallic phases during the phase separation process. The measurements performed indicate a miscibility gap opening discontinuously below $245 \mathrm{~K}$ and rapid freezing of oxygen mobility below $200 \mathrm{~K}$.

At present still growing interest is observed in phenomena related to the phase separation in high- $T_{\mathrm{c}}$ superconductors. This problem has been reviewed recently by Emery and Kivelson [7]. Special workshop devoted to the phase separation was held in Erice in May 1992 (see [13] and [15]). But still many aspects of this subject are not finally clarified, especially the properties of phase domains. Importance of the problem of the phase separation should also be seen in connection with the suggestion [7] that the mechanisms of high- $T_{\mathrm{c}}$ superconductivity might be enhanced by the coupling to dynamic density fluctuations which arise from the frustrated phase separation.

We are going to sum up in this review our experimental data concerning magnetic properties of an oxygenated $\mathrm{La}_{2} \mathrm{CuO}_{4}$ single crystal. For completeness we review also experiments that demonstrate the existence of macroscopic phase separation in photo-doped materials.

\section{Phase separation in $\mathrm{La}_{2} \mathrm{CuO}_{4+\delta}$}

$\mathrm{La}_{2} \mathrm{CuO}_{4}$ is the parent compound for the 2:1:4 copper oxide materials which become superconducting around $40 \mathrm{~K}$. A transition to the superconducting state can be realized either by doping the La sublattice with a divalent alkali-earth element [1] or by introducing extra oxygen [16].

The structure of $\mathrm{La}_{2} \mathrm{CuO}_{4}$ is of $\mathrm{K}_{2} \mathrm{NiF}_{4}$ type and belongs to the space group $\mathrm{Cmca}$ with $\mathrm{Cu}$ ions in $4 \mathrm{a}$ position. The $\mathrm{Cu}$ ions are surrounded by an oxygen octahedron elongated along the $b$ axis. $\mathrm{La}_{2} \mathrm{CuO}_{4}$ is an important example of a Mott-type insulating state possessing long-range canted antiferromagnetic order below a Néel temperature $T_{\mathrm{N}} \simeq 300 \mathrm{~K}$. Oxygen doping has rather strong influence on $T_{\mathrm{N}}$ while its influence on transition temperature $T_{\mathrm{c}}(\approx 40 \mathrm{~K})$ is very small. The fact, that this material has the transition temperature independent of $\delta$, strongly supports the existence of macroscopic phase separation below the room temperature into oxygen rich $\left(\mathrm{La}_{2} \mathrm{CuO}_{4.08}\right.$ ' which becomes superconducting below $\left.40 \mathrm{~K}\right)$ and insulating $\mathrm{La}_{2} \mathrm{CuO}_{4}$ phases. The first direct evidence of the macroscopic phase separation in $\mathrm{La}_{2} \mathrm{CuO}_{4+\delta}$ was given by Jorgensen et al. [6] in 1988 using neutron 
diffraction measurements. They showed that in low temperatures $\mathrm{La}_{2} \mathrm{CuO}_{4+\delta}$ contained two different, but quite similar orthorhombic phases with various oxygen stoichiometry. Note that the two phases belong to the same space group Cmca [17].

Even stronger evidence of phase separation results from microstructure observations performed by Ryder et al. [18] by means of TEM. While cooling from the room temperature to $100 \mathrm{~K}$ they observed the development of a strong contrast between white and black fringes inside each orthorhombic twin domain. This microstructure with periodicity of about $30 \mathrm{~nm}$ is due to the phase separation. The existence of such a mixed-phase microstructure explains the usually observed small volume fractions of superconducting phase in $\mathrm{La}_{2} \mathrm{CuO}_{4+\delta}$.

\section{Magnetic properties of $\mathrm{La}_{2} \mathrm{CuO}_{4+\delta}$ single crystals}

The temperature-dependent magnetic susceptibility (defined as $\chi(T)=$ $M(T) / H$, where $M(T)$ is the magnetization and $H$ is an applied magnetic field) of a $\mathrm{La}_{2} \mathrm{CuO}_{4+\delta}$ single crystal, measured in various magnetic fields applied parallel to the $c$-axis is displayed in Figs. 1 and 2. The samples were measured under slow

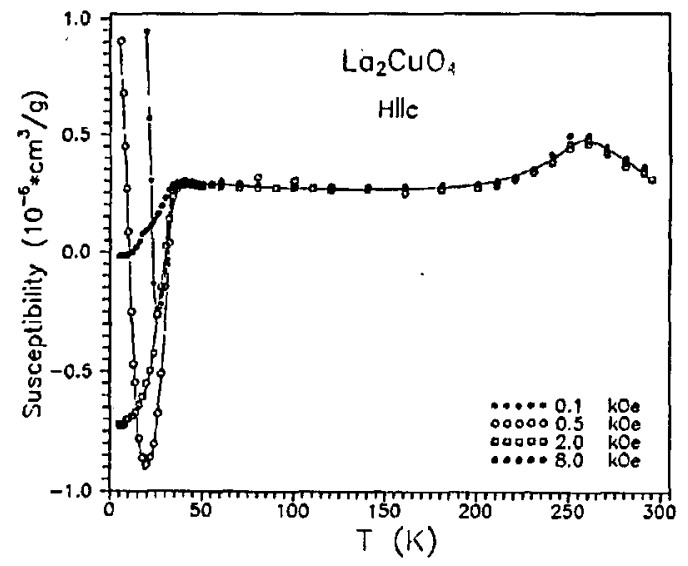

Fig. 1. The temperature dependence of the magnetic susceptibility of $\mathrm{La}_{2} \mathrm{CuO}_{4}$ measured for the sample cooled in various magnetic fields applied parallel to the $c$-axis.

cooling conditions in a given magnetic field $H$. The peak in the magnetization curves at around $260 \mathrm{~K}$ indicates the onset of long-range antiferromagnetic ordering with the Néel temperature $T_{N}$. The value of the Néel temperature does not depend on the magnetic field (up to $H \leq 2 T$; at higher fields the observed peak in $\chi(T)$ shifts to lower temperatures). Below $40 \mathrm{~K}$ (Fig. 2) one can observe a sharp transition from the paramagnetic (or rather antiferromagnetic) to a diamagnetic state. It indicates the presence of superconductivity in the measured samples.

The value of the diamagnetic response depends strongly on the magnitude of the applied field. The minimum of diamagnetic susceptibility, $\chi_{\min }$, is for example, considerably higher for $H=500$ Oe than for $H=100$ Oe. This very unusual behaviour, never observed previously in any superconducting or spin glass systems, 


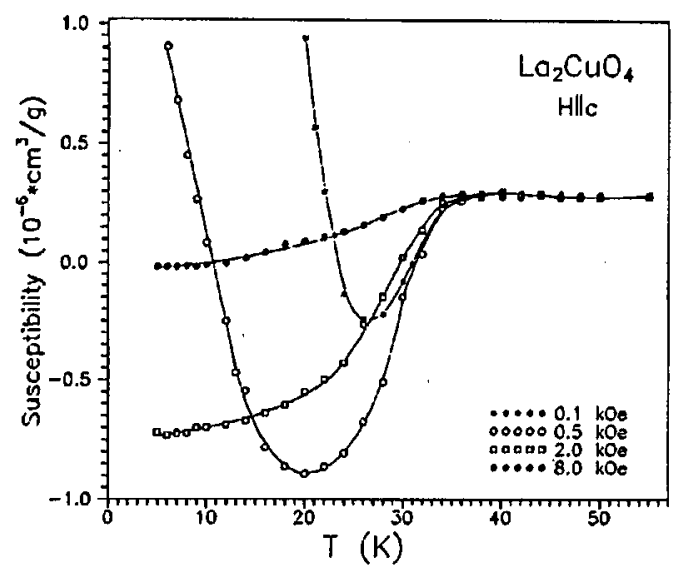

Fig. 2. The low temperature part of the magnetic susceptibility measured under the same conditions as in Fig. 1.

is, in our opinion, the signature of the phase separation existing in our sample. The nonmonotonic $\chi_{\min }(H)$ dependence can be understood while considering the fact that the domain sizes of the superconducting phase are of the same order of the magnitude as the London penetration depth. One should additionally assume that the size of the mixed-phase domains, created during the phase separation process, depends on the magnitude of the applied magnetic field. The effect of magnetic field on diamagnetic response has been also observed in [9]. Kremer et al. [9] have observed that the application of even a weak magnetic field (a few hundred Oe) during the cooling procedure decreases the volume of the superconducting phase.

At low temperatures one can observe (Fig. 2) paramagnetic contribution to the measured susceptibility. This contribution also depends on the magnitude of the applied field and arises due to $\mathrm{Cu}^{2+}$ ions located in domain walls separating phase domains. More or less isolated $\mathrm{Cu}^{2+}$ ions exist mainly in the domain walls where various kind of defects are concentrated leading to breaking the exchange interactions between copper ions. The paramagnetic contribution to magnetic susceptibility is described by the Curie law. This additionally confirms the suggestion concerning the origin of low temperature anomalies observed in $\chi(T, H)$ dependence.

The results presented in Fig. 3 indicate that the phase separation in our samples starts at around $230-240 \mathrm{~K}$. To explain unusual behaviour of $\chi(T)$ for a sample exposed to a 5 Tesla magnetic field during the cooling procedure one should take into account that an applied magnetic field induces the metamagnetic transition to a weak ferromagnetic phase. Below $T_{\mathrm{c}}$ the weak ferromagnetic phase is still stable, even at low fields, due to the magnetic flux trapped by the superconducting domains. A similar effect is not observed for a sample cooled in 3 Tesla magnetic field since this field is not high enough [19] to stabilize the weak ferromagnetic structure down to the superconducting region. The effect of the magnetic flux trapping is also seen in Fig. 4, where a hysteresis loop measured at $10 \mathrm{~K}$ is 


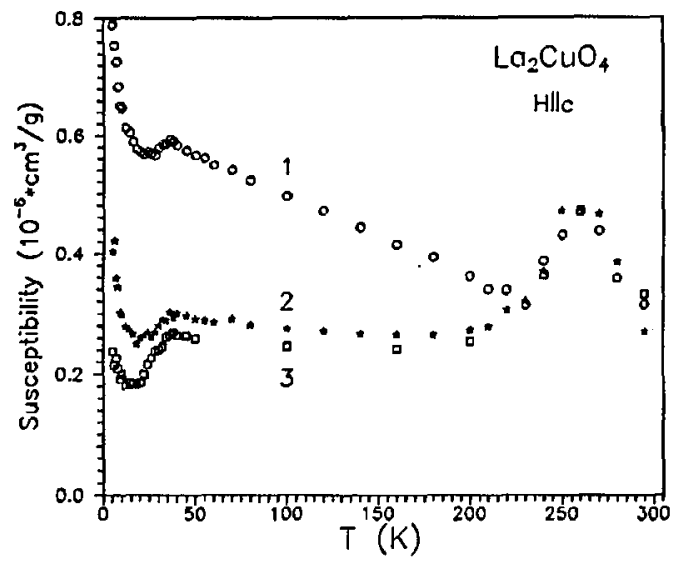

Fig. 3. The temperature dependence of the magnetic susceptibility of $\mathrm{La}_{2} \mathrm{CuO}_{4+\delta}$ measured for the sample cooled from $295 \mathrm{~K}$ down to $5 \mathrm{~K}$ in magnetic fields $H=50 \mathrm{kOe}$ (curve 1), $H=30 \mathrm{kOe}$ (curve 2) and $H=0$ (curve 3 ). Measurements are performed in $H=2$ kOe.
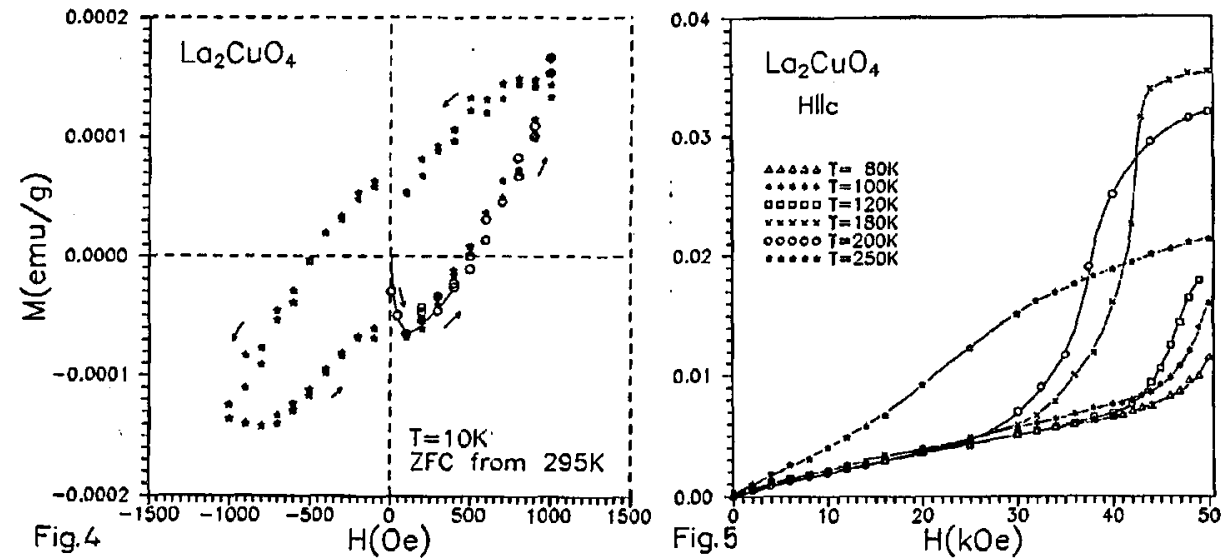

Fig. 4. The hysteresis loop measured for the zero-field cooled sample at $T=10 \mathrm{~K}$.

Fig. 5. Isothermal magnetization vs. magnetic field at various fixed temperatures.

displayed. It can be seen from the $M(H)$ dependence that irreversible effects, due to the flux trapping, are very pronounced. Besides this effect, the hysteresis loop indicates clearly the presence of the large paramagnetic moment due to isolated $\mathrm{Cu}^{2+}$ ions.

Figure 5 presents experimental data confirming the presence of previously mentioned metamagnetic phase transitions in $\mathrm{La}_{2} \mathrm{CuO}_{4+\delta}$ single crystals. The magnetization curves measured at various temperatures indicate a shift of the magnetization jump to lower-field values with increasing temperature. Experimental data presented in Fig. 5 are strongly related to the magnetization measurement 


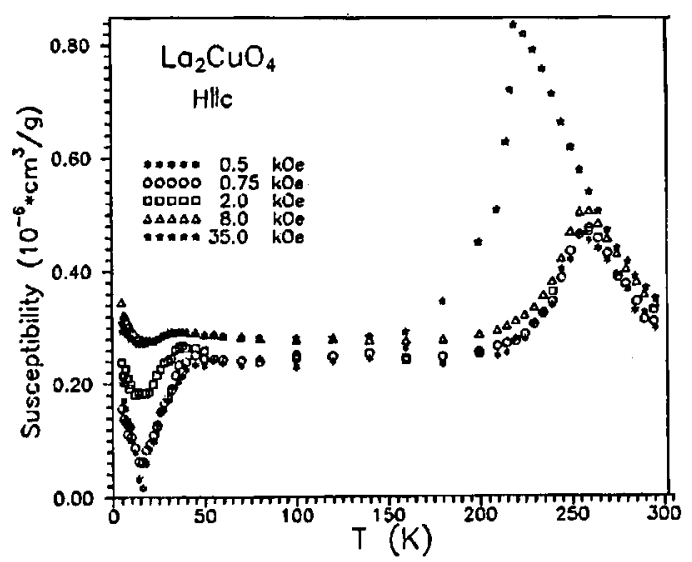

Fig. 6. The temperature dependence of the magnetic susceptibility of $\mathrm{La}_{2} \mathrm{CuO}_{4+6}$ measured in various magnetic fields for the sample cooled in $H=0$ from $295 \mathrm{~K}$ down to $5 \mathrm{~K}$.

performed with various applied fields on a zero-field cooled sample (Fig. 6). A sharp peak observed in $\chi(T)$ curve for a sample measured at $3.5 \mathrm{~T}$ indicates the field-induced (metamagnetic) transition to the weak-ferromagnetic phase. This peak moves down to lower temperatures with increasing field in agreement with experimental data displayed in Fig. 5.

Little is known about the phase separation created with magnetic field applied perpendicularly to the $c$-axis. In Fig. 7 we plot the temperature-dependent magnetic susceptibility measured in $0.2 \mathrm{~T}$ magnetic field applied in the $\mathrm{Cu}-\mathrm{O}$

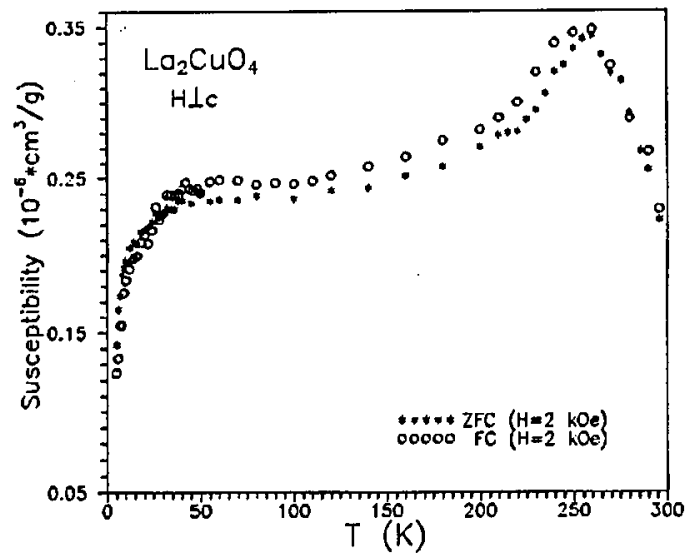

Fig. 7. The magnetic susceptibility of $\mathrm{La}_{2} \mathrm{CuO}_{4+\delta}$ measured in $H=2 \mathrm{kOe}$ applied perpendicular to the $c$-axis for zero-field and field cooled sample. 
plane, for zero-field and field cooling regimes. By comparison the experimental data presented in Fig. 7 with those given in Figs. 1 and 6 it can be concluded that diamagnetic responses for magnetic field applied parallel and perpendicular to the $c$-axis are completely different. It means that the shape and size of phase domains created in both cases are different. In order to clarify this point further experimental investigations are required.

\section{4. $\mathrm{EPR}$ of $\mathrm{Cu}^{2+}$ ions in $\mathrm{La}_{2} \mathrm{CuO}_{4+\delta}$}

There are many observations of EPR spectra for high- $T_{\mathrm{c}}$ superconductors but most of them are attributed to a foreign phase [20]. In spite of that it seems that under some conditions an EPR signal is to be observed in single crystals as well as in ceramic samples. According to [21] EPR signals arise from the transition regions between orthorhombic and tetragonal phases. It suggests that the domain walls separating metallic and dielectric phases in $\mathrm{La}_{2} \mathrm{CuO}_{4+\delta}$ exhibiting the macroscopic phase separation should also be considered as the probable source of the EPR signal. Figure 8 presents [22] the EPR spectrum observed in specimens which had

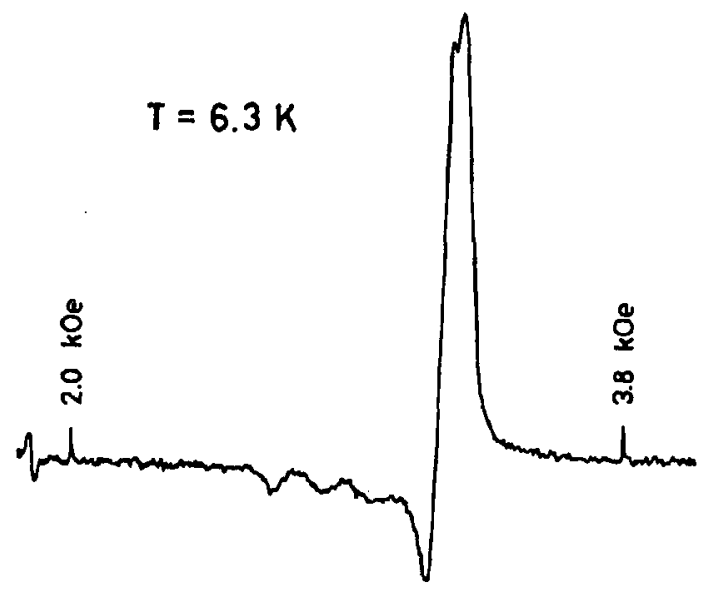

Fig. 8. The $\mathrm{EPR}$ spectrum of $\mathrm{La}_{2} \mathrm{CuO}_{4+\delta}$ measured at $T=5 \mathrm{~K}$.

been used previously to study the phase separation effects in $\mathrm{La}_{2} \mathrm{CuO}_{4+\delta}$. To obtain high enough intensity of the EPR lines, specimens are in the powder form obtained by careful crushing of single crystals. The EPR spectrum has been recorded under identical conditions, under which the phase separation was observed. What is fascinating in this spectrum is the observation of the hyperfine structure, never been observed previously in high- $T_{\mathrm{c}}$ superconductors. This result confirms, without any doubt, that the EPR signal is due to the $\mathrm{Cu}^{2+}$ ions. Very narrow, symmetrical lines cannot arise from the domain walls, because these are characterized by various types of lattice defects leading to broadening of the resonance lines. We suppose that the observed EPR spectrum should be attributed to $\mathrm{Cu}^{2+}$ localized moments 
in the metal. Since the characteristic size of the metallic phase domains in phase separated samples is much smaller than the skin-depth (estimated to be of the order of $10 \mu \mathrm{m}$ ) one should expect to observe symmetrical EPR lines in accordance with Fig. 8. Further confirmation of the proposed origin of the EPR signal is the strong correlation observed between diamagnetic response (determined by the number and size of the phase domains) and the intensity of the EPR spectrum. It seems that the EPR signal from ions inside the domain walls is too weak to be observed.

Note that the EPR spectrum reported in Ref. [23] has been observed in samples prepared by the fast quenched procedure which suppresses the phase separation.

\section{Phase separation in photo-doped cuprates}

At present there is still growing interest in achieving metallic state (and superconductivity) starting from the insulating (antiferromagnetic) parent material through carrier generation directly onto the $\mathrm{CuO}_{2}$ plane by photoexcitation (photodoping) (see review paper [24] and references therein). The main advantage of photodoping compared with chemical doping is that photodoping allows one to change the carrier density without any changes in chemical composition and lattice structure of the parent material. In the photo-excitation experiments a parent material is exposed to high intensity laser radiation with photon energies in excess to the insulating gap. The photo-generated holes are located in $\mathrm{CuO}_{2}$ planes and migrate to form droplets of metallic carrier concentration. These metallic droplets formed in photo-doped materials are responsible for the appearance in optical spectrum some features characteristic of metallic phase (note that the concentration of photo-generated carriers is less than $1 \%$ [7] and uniformly distributed photo-generated holes at such densities could not cause any observable change in optical spectrum). Experimental data concerning optical spectrum as well as photoconductivity imply the electronic phase separation in photo-doped cuprates.

\section{Conclusions}

We have shown that many of the unusual features of the $\mathrm{La}_{2} \mathrm{CuO}_{4+\delta}$ superconductor derive from the fact that this material exhibits the macroscopic phase separation. The fingerprint of the macroscopic phase separation is the presence, in some temperature range, of both superconductivity and a long-range antiferromagnetic ordering. Properties of the mixed-phase state depend strongly on the shape and size of the phase domains formed during the phase separation process. In the case of photo-doped cuprates observed, the phase separation is driven electronically. It is suggested that the driving force for the phase separation in $\mathrm{La}_{2} \mathrm{CuO}_{4+\delta}$ is a combination of the chemical and electronic mechanisms.

This paper has been supported in part by the Committee for Scientific Research under contract No. 204849101. 


\section{References}

[1] J.G. Bednorz, K.A. Müller, Z. Phys. B 64, 189 (1986).

[2] P.B. Vischer, Phys. Rev. B 10, 943 (1974).

[3] V.J. Emery, S.A. Kivelson, H.Q. Lin, Phys. Rev. Lett. 64, 475 (1990).

[4] S.A. Kivelson, V.J. Emery, H.Q. Lin, Phys. Rev. B 42, 6523 (1990).

[5] M. Grilli, C. Raimondi, C. Castellani, C. DiCastro, G. Kotliar, Phys. Rev. Lett. 67, 259 (1991).

[6] J.D. Jorgensen, B. Dabrowski, P. Shiyou, D.G. Hinks, L. Soderholm, B. Morosin, J.E. Schirber, E.L. Venturini, D.S. Ginley, Phys. Rev. B 38, 11337 (1988).

[7] V.J. Emery, S.A. Kivelson, Physica C 209, 597 (1993).

[8] G. Yu, C.H. Lee, A.J. Heeger, N. Herron, E.M. McCarron, L. Cong, G.C. Spalding, C.A. Nordman, A.M. Goldman, Phys. Rev. B 45, 4964 (1992).

[9] R.K. Kremer, E. Sigmund, V. Hizhnyakov, F. Hentsch, A. Simon, K.A. Müller, M. Mehring, Z. Phys. B 86, 319 (1992).

[10] R.K. Kremer, V. Hizhnyakov, E. Sigmund, A. Simon, K.A. Müller, Z. Phys. B 91, 169 (1993).

[11] V. Hizhnyakov, E. Sigmund, Physica C 156, 655 (1988).

[12] J.H. Cho, F.C. Chou, D.C. Johuston, Phys. Rev. Lett. 70, 222 (1993).

[13] M. Mehring, M. Baehr, P. Gergen, J. Gross, C. Kessler, W. Winzek, in: Phase Separation in Cuprate Superconductors, Eds. K.A. Müller, G. Benedek, World Scientific, Singapore 1993, p. 67.

[14] E.T. Ahrens, A.P. Reyes, P.C. Hammel, J.D. Thompson, P.C. Canfield, Z. Fisk, J.E. Schirber, Physica $C$ 212, 317 (1993).

[15] P.C. Hammel, E.T. Ahrens, A.P. Reyes, J.D. Thompson, Z. Fisk, P.C. Canfield, J.E. Schirber, in: Phase Separation in Cuprate Superconductors, Eds. K.A. Müller, G. Benedek, World Scientific, Singapore 1993, p. 139.

[16] J.E. Schirber, B. Morosin, R.M. Merill, P.F. Hlava, E.L. Venturini, J.F. Kwak, P.J. Nigrey, R.J. Baughman, P.S. Ginley, Physica C 152, 121 (1988).

[17] C. Chaillout, J. Chenavas, S.W. Cheong, Z. Fisk, M. Marezio, B. Morosin, J.E. Schirber, Physica C 170, 87 (1990).

[18] J. Ryder, P.A. Midgley, R. Exley, R.J. Beynon, D.L. Yates, L. Afalfiz, J.A. Wilson, Physica C 173, 9 (1991).

[19] S.W. Cheong, J.D. Thompson, Z. Fisk, Phys. Rev. B 39, 4395 (1989).

[20] D.C. Vier, S.B. Oseroff, C.T. Salling, J.F. Smith, S. Schultz, Y. Dalichough, B.W. Lee, M.B. Maple, Z. Fisk, J.D. Thompson, Phys. Rev. B 36, 8888 (1987).

[21] I.A. Garifullin, N.N. Garifyanov, N.E. Alekseevskii, S.F. Kim, Physica $C$ 179, 9 (1991).

[22] S. Piechota, H. Szymczak, R. Szymczak, in preparation.

[23] G. Wübbeler, O.F. Schirmer, Phys. Status Solidi B 174, K21 (1992). 